\title{
MODERNISATION OF BOILER UNIT AUTOMATION SYSTEM AT ACTING POWER STATION
}

\author{
Aleksei A. Abramovskikh ${ }^{1,{ }^{*}, \text { Ulyana A. Ulyanova }}{ }^{1}$, Sergey V. Lavrinenko ${ }^{1}$, Anastasiya A. \\ Matveeva $^{1}$, and Dmitry Malishev ${ }^{1}$ \\ ${ }^{1}$ Tomsk Polytechnic University, 634050, Tomsk, Russia
}

\begin{abstract}
The article deals with the modernization process of automated control system of operating process of boiler unit at South Kuzbas hydro electric power station. The boiler unit has been modernized through the installation of automated control system and control on hierarchical arrangement consisting of the levels where the tasks of input and ouput control signals are distributed. Controllers produced by Ram Company and sensors produced by Elemer company were used for modernization of boiler unit. Automatic and process control was made on the base of MicroSCADA software. It has been currently estimated that the use of automation system will result in decrease in fuel consumption by $1 \%$ and reduce nitrogen oxide and carbon monoxide emission by $3 \%$. System payback time would be no more than two years.
\end{abstract}

\section{Introduction}

Constant energy resource price rise for energy resources in particular for fossil fuel is one of the main problems in modern power industry [1]. Severe climatic environment in Russia has become one of the reasons of extensive development of boiler systems with different fuel types including alternative fuel [2, 3]. They provide essential conditions for accommodation facilities and business. Therefore, technical problem solving connected with effective boiler operation control and its development is one of the most important ways of science and technology development [4].

High wear of public infrastructure is a current problem for many regions in Russia [5]. The work on heat networks and boiler systems modernization does not only allow to prevent areal communal fail but also leads to the increase of energy efficiency - old equipment is replaced with the new one with improved performance [6-7]. According to experts estimates there will be collapse in the Communal Service Market without modernization of local boiler plants. First of all many of them will break down, and no one operation organization will take on running worn-out equipment. Secondly a lot of people will have to use furnace heat, that is go back in time several decades. Modernization of boiler plants also implies not only the change over of equipment but also the parts of heat

*Corresponding author: aleksey82@,tpu.ru 
networks. All this leads to reduction of heat losses, and as a consequence keeps the amount charges down [8]. This question is also relevant in the context of atomic power stations where special attention is paid to the control systems parameters [9]. For nuclear energy modeling of different processes is widely used for security purposes [10] with further analysis of more optimal adjustments [11].

\section{Objectives of the research}

One of the major challenge associated with operation of boiler unit is to ensure balance between generated and consumed energy. In its turn processes of vaporization and energy transmission in boiler unit are definitely connected with the amount of flow of working medium and heat storage medium. Burn rate, efficiency and fuel burning stability depends on the air supply and air distribution between the fuel particles. It is conventionally accepted to divide the process of fuel consumption into three stages: breakdown, burning and reburning $[12,13]$. These stages happen consistently, sometimes with overlap. Burning calculation means the determination of air amount in cubic meters which is necessary for burning of unit of mass or the volume of fuel amount and the composition of heat balance and burning temperature test [14]. Nowadays the equipment is out dated and is not maintainable. The majority of control circuits are switched to manual mode.

The aim of modernization of boiler unit SB-10 (steam boiler) at joint stock company South Kuzbas hydro electric power station is to remove defects of the existing system and to provide the increase of fuel consumption rate on steam generation and hot water for consumers. The aim of this boiler unit is gathering information about process value and working conditions, operation of working conditions and also archiving of measured parameters values and graphing its changes.

Power regulation of boiler units and pressure control in boiler drum is in maintenance of balance between vapor extraction and water supply. The level of water in boiler drum is the key parameter that characterizes the balance. The reliability of boiler unit work depends on the quality of level control. When the pressure gets high, the decrease below the acceptable limits can lead to circulation loss in water tubes. It can result in temperature increase of pipe walls and their over burn.

The increase can also result in emergencies, because water flow in steam heater can happen and it breaks down. The highest demands are made on accuracy of maintenance of required level. Feed water control is defined by the equality of water supply. It is necessary to provide boiler with equal water supply because frequent and deep change in feed water flow can result in serious thermal tensions in the metal of economizer.

Boiler drums with natural circulation are intrinsic to some considerable storage capacitance which manifests itself in transient states. If in transient mode the level of water in boiler drum is determined by the condition of burden material balance then in transient states big amount of insurges influences level position.

The main insurges are the change of feed water flow, change of boiler output if consumer load is changed and change of firebox load, the change of feed water flow temperature.

The regulation of ratio fuel-air is required both from physical and economical point of view. Fuel burning is known to be the most important processes that takes place in boiler plant. The chemical part of fuel burning is oxidation reaction of fuel elements by oxygen molecules. The oxygen is used for burning. The air to firebox is supplied in a particular proportion with fuel by means of forced flow-fan. The ratio fuel - air is approximately $1: 1.3$. In case of lack of air incomplete fuel burning takes place. Unburned fuel is released into the atmosphere that is neither economically nor ecologically acceptable. The cooling of firebox will be in furnace chamber in case of extra fuel, but the fuel will burn out. In this 
case the rests of air will form nitrogen dioxide which is impossible from ecological point of view, because this combination is dangerous for people and environment. Parameters alarm and protection working on boiler shut down are essential because operator is unable to keep track of all parameters of working boiler. An emergency situation can result from this. On running the water from out of boiler drum the level of water is decreased in it, as a consequence circulation is lost and pipe over burn of bottom screen happens. Active protection prevents generator breakdown. On decreasing generator load, burn rate in firebox decreases. Burning becomes non-steady and can stop. There is protection of flame distinguishing.

In boiler unit SB-10 (Fig. 1) the burning process takes place in furnace chamber where the feedwater goes down the pipes, saturated steam travels through the waterwall pipes. Combustion products, air - fuel come on furnace chamber through the burners. The boiler unit contains multiloop superheater of a mixed type with cross flow of coil with sweep of gases. Superheater is used for transformation of saturated steam into hot vapour [15]. Economizer is a continuous loop type device of a boiling type, a smooth pipe battery with horizontal position of coils with cross flow of sweep of gases. Economizer is aimed at preheating of feed water. It consists of two blocks. The second block of air heater is located between the blocks of economizer which also consists of two blocks. Air heater is a pipe four-way type, with longitudinal sweep of gases. Air heater is used to heat the air which is meant for combustion.

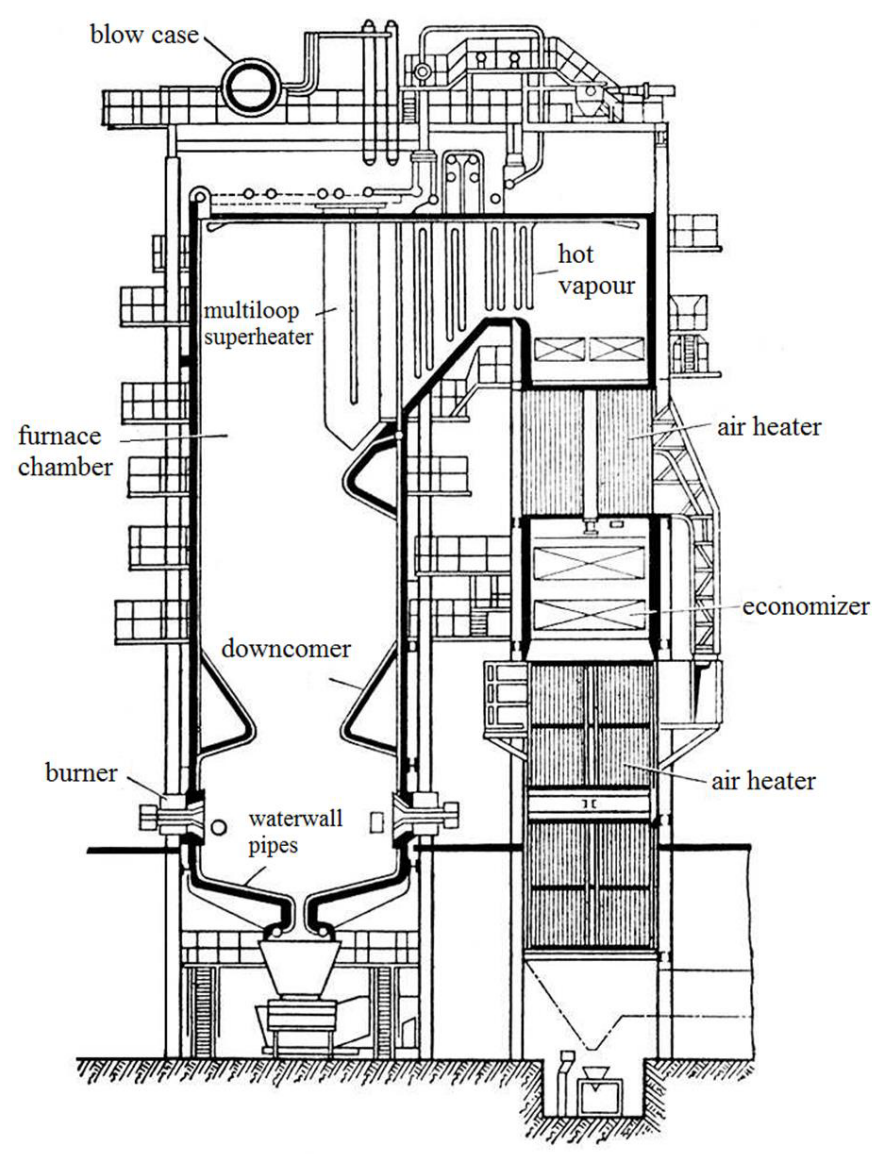

Fig. 1. Boiler PK-10 in elevation. 


\section{Methods of research and results}

Modernization of SCADA boiler unit was made on hierarchical arrangement consisting of three levels of automatic performance:

1 level - "Field level of the system" (basic automatic devices - fuel gages, pressure and temperature transmitters, power units). They provide measurement of input and output signals of boiler unit, control of regulating parts and information transmission to the next system level.

2 level - "Lower level of the system" (programmable controller with input/output devices) is connected with the field level of the system with the use cables.

3 level - "High level of the system" - computer-based working stations of operators with software tools including SCADA programs and standard interface.

SCADA-system means specialized software program implementing man-machine interface [16].

Controllers produced by Ram company were used for modernization of boiler unit. These controllers have proved to be the best on the Russian market as quality price indicator.

Fuel gages, pressure and temperature transmitters, power units that have been utilized in research are produced by Elemer company. These devices are used in parameter control system of boiler unit and they are widely recognized as reliable devices. Micro-SCADA is software program SCADA, that implements automatic operation and control of technological process and specializes in automatic performance in certain field [8]. The work of boiler units, the key process conditions, alarm messages can be tracked on the monitor schematically (fig. 2,3).Visualization system besides displaying the main parameters such as pressure, temperature, level, fuel consumption, regulatory body statue and current load of electric devices makes measurement of boiler capacity in units of evaporation, fuel rate and relative hour fuel consumption rate. Mnemonic scheme in the form of pictograms shows whether actuating units are in manual or automatic mode.

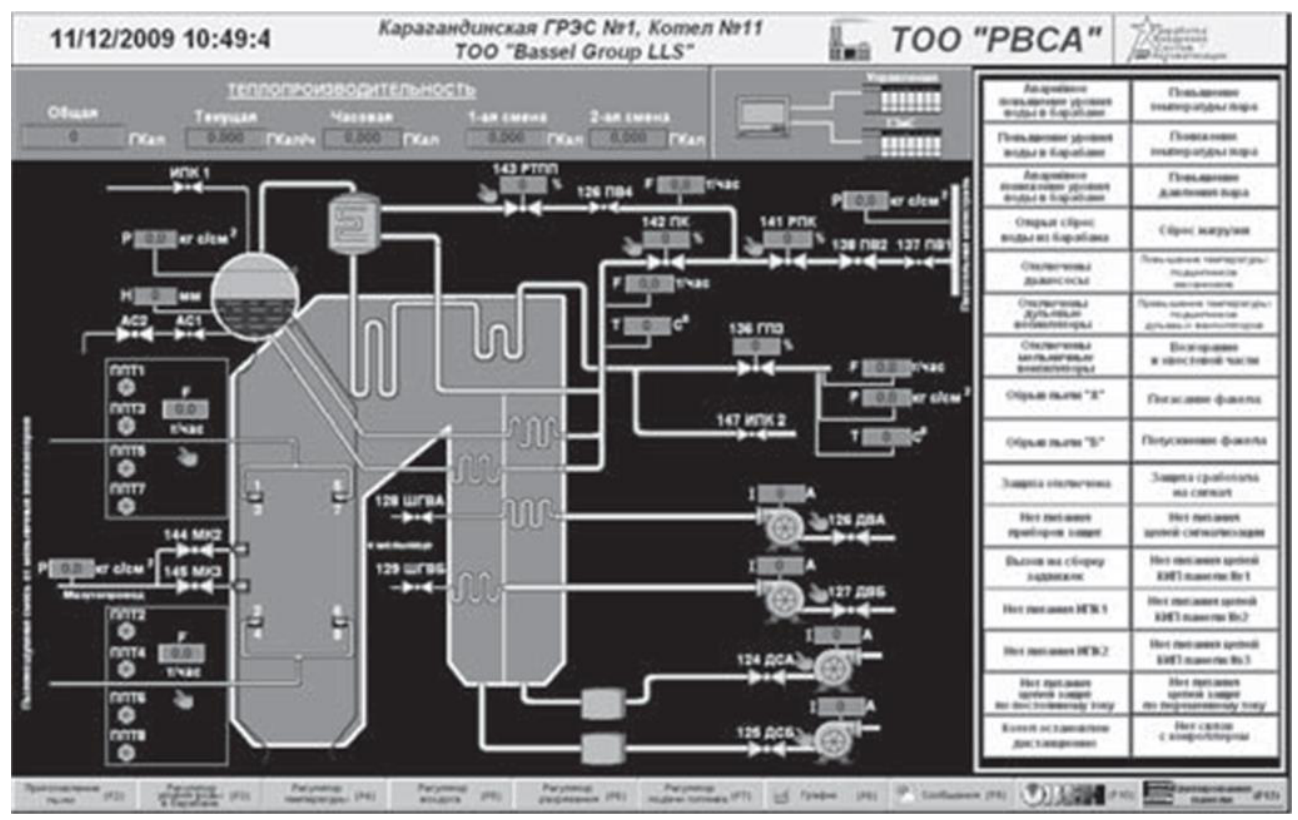

Fig. 2. Mnemonic scheme of boiler unit. 


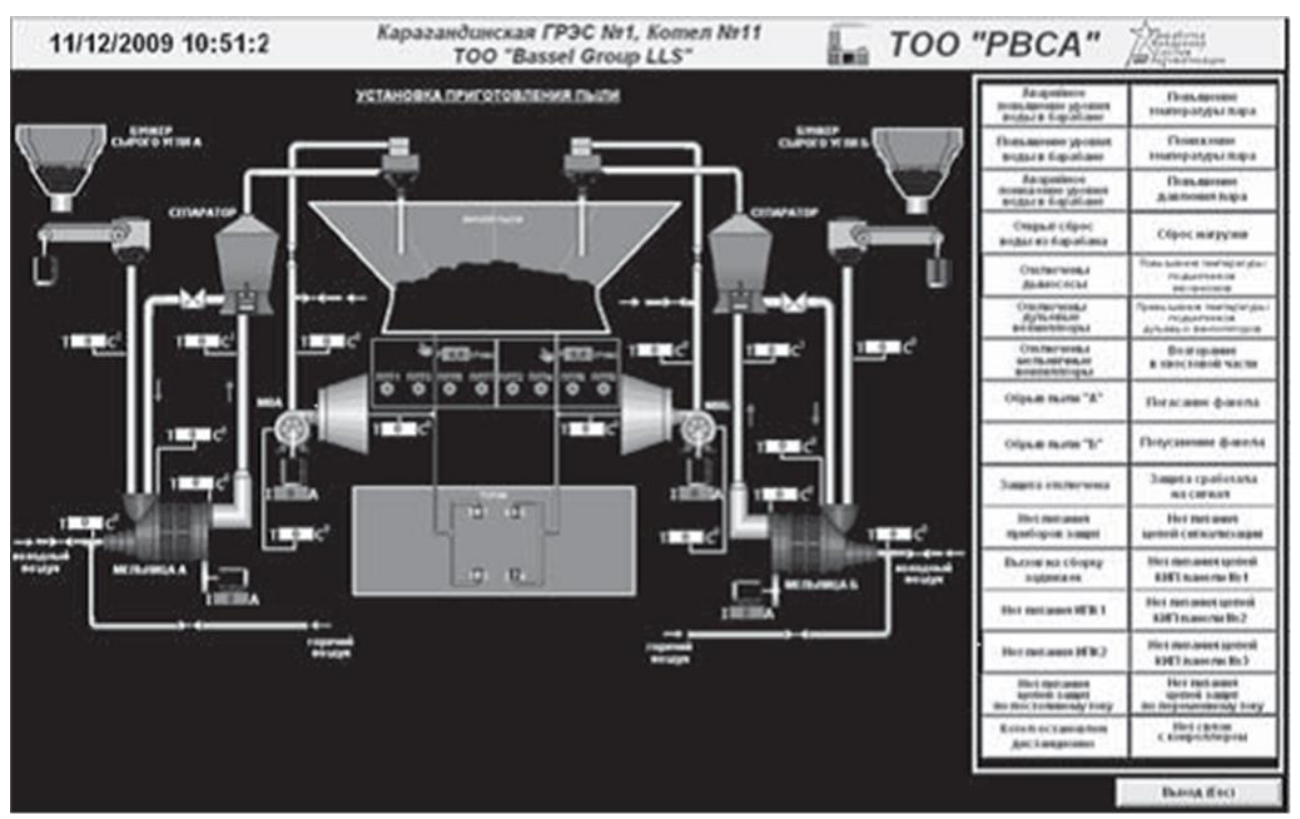

Fig. 3. Mnemonic scheme of Pulverized coal system.

Emergency control automatics provides automatic stop of boiler in critical operations when some parameter exceeds the value by conditions of process safety or possible equipment breakdown. The operation of alarm system is accompanied by audio and light alarm, display of messages on to the primary and secondary stations of operator. The system shows the reasons of accidental situation. All events are recorded on the message window in chronometrical sequence with recording the time of occurrence, acknowledgement and signal loss.

\section{Conclusion}

Functional structure of improved system of boiler unit control has been developed. All technical facilities have been chosen out of optimal ratio price-quality. Improvement of process automation system of the process of boiler unit operation PK-10 a joint stock company South Kuzbas hydro electric power station will result in decrease in fuel consumption by $1 \%$ and reduce nitrogen oxide and carbon monoxide emission by $3 \%$. System payback time would be no more than two years.

The study was realized in "National research Tomsk polytechnic university" in framework of federal target program "Research and development in prior directions of scientifictechnological complex development in Russia in 2014-2020 year", unique identifier of $R \& D$ project RFMEFI58114X0001.

\section{References}

1. D. A. Pavlov, E. N. Semikova, Modern high-end technologies, 8-2, 309 (2013)

2. A. N. Golovanov, A. S. Yakimov, A. A. Abramovskikh, Y. R. Sukov, Thermophysics and Aeromechanics, 15, 4, 651 (2008). 
3. A. M. Grishin, A. N. Golovanov, Ya. V. Sukov, A. A. Abramovskikh, Journal of Engineering Physics and Thermo-physics, 80, 6, 1154 (2007)

4. A. A. Silaev, S. N. Zadvorskiy, Bulleting of Volgograd state technical university, 13 (177), 141 (2015)

5. A. Zenkov, S. Yankovsky, A. Matveeva, S. Lavrinenko, A. Gromov, MATEC Web of Conferences, 72, 01131 (2016)

6. E. Pavelyev, S. Lavrinenko, MATEC Web of Conferences, 72, 01085 (2016)

7. K. Savina, A. Abramovskih, V. Haymin, S. Lavrinenko, MATEC Web of Conferences, 72, 01042 (2016)

8. V. Martov, Sanitary engineering, heating and conditioning, 7 (151), 94 (2014)

9. A. V. Vorobiev, A. M. Antonova, M. P. Vinogradov, EPJ Web of Conferences, 110, 01053 (2016)

10. A. M. Antonova, A. V. Vorobiev, A. S. Orlov, MATEC Web of Conferences, 23, 01069 (2015)

11. A. Uvarov, A. Antonova, A. Vorobjev, MATEC Web of Conferences, 37, 01062 (2015)

12. N. Galashov, S. Tsibulskii, A. Matveev, V. Masjuk, EPJ Web of Conferences, 110, 01019 (2016)

13. A. A. Somov, D. A. Mel'Nikov, D. V. Gvozdjakov, V. E. Gubin, Yu. S. Borovikov, A. S. Matveev, MATEC Web of Conferences, 37, 01054 (2015)

14. M. I. Reznikov, Yu. M. Lipov, Boiler units of power stations (Mir Publishers Moscow) (1989)

15. V. G. Aleksandrov, Steam boilers of low and average power (1966)

16. Operating Manual TRACE MODE 6 u T-FACTORY, (Москва 2011) 